\title{
NMR in Ferromagnetic Manganites
}

\author{
P. NOVÁK
}

Institute of Physics, Academy of Sciences of the Czech Republic

Cukrovarnická 10, 16253 Praha 6, Czech Republic

The conditions for observing NMR on the ${ }^{55} \mathrm{Mn}$ nuclei in the ferromagnetic manganites are specified and the information which may be extracted from this NMR are discussed. As examples, the study of the order of the magnetic phase transition and the coexistence of different ferromagnetic phases in the ferromagnetic manganites are presented.

PACS numbers: 71.15.Mb, 76.30.Fc, 71.70.Jp

\section{Introduction}

Important question behind the complex physics of the ferromagnetic manganites is the electronic structure of the manganese ions and the related problem of the nature of charge carriers - are the charge carriers localized or itinerant, do they coexist with localized $\mathrm{Mn}^{3+}$ and $\mathrm{Mn}^{4+}$ ions, is a system single phase or several different magnetic phases occur. Nuclear magnetic resonance is a suitable tool to address these problems as it probes the manganese ions locally and a wealth of information may be obtained concerning the local magnetic fields and the nuclear spin dynamics. Yet a caution is needed when drawing the conclusion and the results of the analysis of NMR should be combined and/or confronted with the conclusions obtained by other experimental methods. All results described in this contribution were obtained by the two-pulse spin-echo method and thus we present first a brief description of this method and discuss the quantities which may be deduced from the analysis of the results. In the third section the usefulness of combining NMR with the magnetic moment measurement is illustrated on the study of the nature of the magnetic transition. Finally, we address the problem of the coexistence of different ferromagnetic phases in ferromagnetic, metallic-like manganites.

\section{Spin-echo method}

In the two-pulse spin-echo method the nuclear spins are aligned along a static, external and/or exchange magnetic field. An exciting radiofrequency pulse 
is applied, which rotates the nuclear magnetization perpendicular to the direction of the static field. It then begins to rotate with the Larmor frequency. Because of the inhomogeneity present in the sample there always exists a spread of this frequency. After a time interval $\tau$ a refocusing pulse, turning the magnetization by $\pi$ is applied. A spin-echo then appears after waiting for another time interval $\tau$, when the nuclear magnetic moments merge.

There are several quantities which could be extracted from such measurement and valuable information may be obtained by analysing them. Below we briefly describe these quantities with a special respect paid to the NMR of ${ }^{55} \mathrm{Mn}$ in manganites.

\subsection{Resonance frequency}

If the nucleus does not possess a quadrupolar moment or if the quadrupolar interaction can be neglected, the nuclear magnetic resonance frequency $f$ is connected with the static magnetic field $B_{\mathrm{N}}$ on the nuclei by

$$
f=g_{\mathrm{N}} \mu_{\mathrm{N}} B_{\mathrm{N}} / h
$$

where $g_{\mathrm{N}}, \mu_{\mathrm{N}}$, h are nuclear $g$ factor, nuclear magneton, and the Planck constant, respectively. The ${ }^{55} \mathrm{Mn}$ nuclei has a nuclear spin $I=5 / 2$ and $g_{\mathrm{N}} \mu_{\mathrm{N}} / h=$ $10.573 \mathrm{MHz} / \mathrm{T}$. The field $\boldsymbol{B}_{\mathrm{N}}$ is a sum of the external magnetic field $\boldsymbol{B}_{\text {ext }}$ and the hyperfine field $\boldsymbol{B}_{\mathrm{hf}}$ arising from the on-site magnetic dipolar interaction between the nuclear and electronic magnetic moments

$$
\boldsymbol{B}_{\mathrm{N}}=\boldsymbol{B}_{\mathrm{ext}}+\boldsymbol{B}_{\mathrm{hf}} .
$$

In principle the magnetic dipolar field $\boldsymbol{B}_{\text {dip }}$, originating from all other magnetic moments present in the system, should be added. In ferromagnetic manganites, however, $\boldsymbol{B}_{\text {dip }}$ is much smaller than $\boldsymbol{B}_{\mathrm{hf}}$ and it can be thus safely neglected.

The magnetic hyperfine interaction and corresponding $\boldsymbol{B}_{\mathrm{hf}}$ may be written as

$$
\mathcal{H}_{\mathrm{hf}}=\boldsymbol{I} \hat{A} \boldsymbol{S}, \quad \boldsymbol{B}_{\mathrm{hf}}=\frac{1}{g_{\mathrm{N}} \mu_{\mathrm{N}}} \hat{A}\langle\boldsymbol{S}\rangle
$$

where $\boldsymbol{S}$ is the electronic spin, $\hat{A}$ is the hyperfine coupling tensor. If the symmetry of the site is cubic or if the orbital momentum of the electronic state may be neglected the hyperfine coupling tensor reduces to a constant and

$$
\boldsymbol{B}_{\mathrm{hf}}=\frac{A}{g_{\mathrm{N}} \mu_{\mathrm{N}}}\langle\boldsymbol{S}\rangle \text {. }
$$

The temperature dependence of the hyperfine coupling constant $A$ is usually weak and thus the temperature dependences of $B_{\mathrm{hf}}$ and the spin magnetic moment of the ion coincide.

In manganites the $\mathrm{Mn}$ ions are octahedrally coordinated. The ground state of the $\mathrm{Mn}^{4+}$ ion has the electronic spin $S=3 / 2$ and in the octahedral crystal field it is orbital singlet. To a good approximation hyperfine tensor then reduces 
to a constant even in a distorted octahedron with the typical value of $B_{\mathrm{hf}}\left(\mathrm{Mn}^{4+}\right)$ at $0 \mathrm{~K}$ around $30 \mathrm{~T}$ (corresponding $f\left(\mathrm{Mn}^{4+}\right) \approx 32 \mathrm{MHz}$ ). For $\mathrm{Mn}^{3+}$ ion $S=2$ and in a regular octahedron the ground state is orbital doublet ${ }^{5} E_{g}$ with a frozen orbital momentum. Due to the presence of excited triplet states ${ }^{5} T_{2 g},{ }^{3} T_{1 g}$ the orbital momentum is not completely frozen and, in a distorted environment, $\hat{A}$ may be anisotropic. To our knowledge no reliable information on this anisotropy in manganites may be found in the literature, though the NMR results in the manganese ferrite [1] suggest that it can be large. In manganites $B_{\mathrm{hf}}\left(\mathrm{Mn}^{3+}\right)$ at $0 \mathrm{~K}$ is typically around $40 \mathrm{~T}\left(f\left(\mathrm{Mn}^{3+}\right) \approx 420 \mathrm{MHz}\right)$.

In the manganites with a mixed valence of $\mathrm{Mn}$ the form of the ${ }^{55} \mathrm{Mn}$ NMR spectra depends on the frequency $f_{\text {hop }}$ with which the electron hole is hopping between the $\mathrm{Mn}$ sites. If $f_{\text {hop }}$ is much smaller than the difference $\Delta f=f\left(\mathrm{Mn}^{3+}\right)-$ $f\left(\mathrm{Mn}^{4+}\right)$, NMR spectrum consists of two distinct $\mathrm{Mn}^{3+}$ and $\mathrm{Mn}^{4+}$ lines. In the opposite limit $f_{\text {hop }} \gg \Delta f$ the NMR experiment sees an average valence state of $\mathrm{Mn}$ and a single "motionally narrowed" NMR line is observed. The frequency of this line equals to $\left[f\left(\mathrm{Mn}^{3+}\right)-f\left(\mathrm{Mn}^{4+}\right)\right] / 2$ if the hopping is between the two sites only, while it coincides with the center of gravity of $\mathrm{Mn}^{3+}$ and $\mathrm{Mn}^{4+}$ lines when the hopping involves a large number of the sites. Let us note that $\Delta f \approx$ $100 \mathrm{MHz}$ is much smaller than the hopping frequency necessary for the system to be classified as metallic and thus the observation of a motionally narrowed spectrum is necessary, but not sufficient condition for the system to be metallic.

\subsection{Relaxation of nuclear magnetization}

After the excitation $\pi / 2$ pulse is applied, the component of the nuclear magnetization perpendicular to the static field begins to decrease exponentially $m_{\perp} \sim \exp \left(-t / T_{2}\right)$, where $T_{2}$ is the spin-spin relaxation time. Simultaneously the parallel component approaches exponentially its equilibrium value $m_{\|}=$ $m_{\text {eq }}\left[1-\exp \left(-t / T_{1}\right)\right], T_{1}$ being the spin-lattice relaxation time. $T_{2}$ is always smaller than $2 T_{1}$, in many ferromagnetic manganites being shorter by an order of magnitude. During the time interval $\tau$ between the exciting and refocusing pulses $m_{\perp}$ partially decays and, as a consequence, the amplitude $\mathcal{A}$ of the spin-echo decreases with decreasing $T_{2}$ :

$$
\mathcal{A}(\tau)=\mathcal{A}(0) \exp \left(-2 \tau / T_{2}\right)
$$

When discussing the amplitude of the spin-echo it is thus important to extrapolate its value to $\tau \rightarrow 0$. Failure to do that might lead to a misconception (see [2] for a detailed discussion). In Fig. 1a the NMR spectra of ${ }^{55} \mathrm{Mn}$ in $\mathrm{La}_{0.7} \mathrm{Ca}_{0.3} \mathrm{MnO}_{3}$ at temperature $77 \mathrm{~K}$, taken with different $\tau$, are displayed. For $\tau=70 \mu$ s the spectrum consists of two peaks which could be attributed to two different Mn centers. This would be an erroneous conclusion, however, as for shorter $\tau$ the structure disappears and the true NMR spectrum is a single, motionally 
narrowed line. As seen in Fig. 1b the "structure" of $\tau=70 \mu$ s spectrum is caused by the fact that the relaxation rate depends on the frequency, it is maximal at the center of the line and the fast relaxation of corresponding spins reduces the spin-echo signal ( $c f$. Eq. (5)). This behavior is typical of the Suhl-Nakamura (SN) mechanism of the relaxation [3], effectiveness of which increases with increasing number of resonating nuclear spins. We also note that the SN mechanism strongly depends on the temperature - it decreases as the temperature is raised.

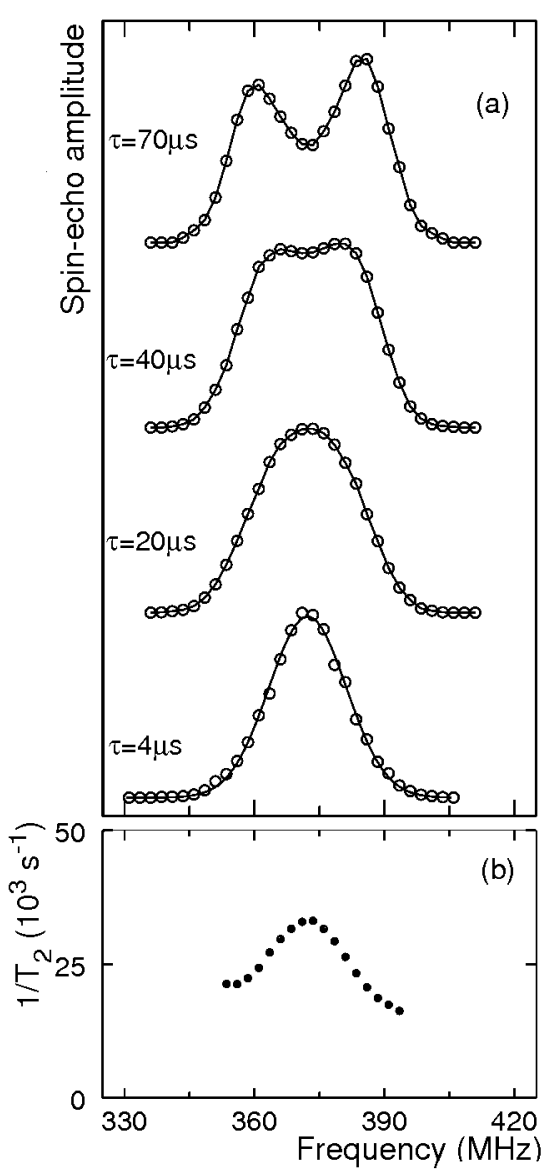

Fig. 1. $\mathrm{La}_{0.7} \mathrm{Ca}_{0.3} \mathrm{MnO}_{3}$ polycrystal at temperature $77 \mathrm{~K}$. (a) NMR spectrum of ${ }^{55} \mathrm{Mn}$ for different values of $\tau$. (b) Spin-spin relaxation rate $T_{2}^{-1}$ as a function of frequency.

In a number of ferromagnetic manganites with the metallic-like conductivity the relaxation rates $T_{2}^{-1}$ and $T_{1}^{-1}$ depend exponentially on the temperature $[4,5]$. This can be understood as caused by the hopping of the electron holes that induces fluctuation of the hyperfine field on the Mn nuclei. As a function of the characteristic time $\tau_{\text {hop }}$ of the hopping process, the relaxation rate attains a maximum for 
$f \tau_{\text {hop }}=1:$

$$
T_{1}^{-1} \sim \frac{f \tau_{\text {hop }}}{1+\left(f \tau_{\text {hop }}\right)^{2}} .
$$

For the motionally narrowed lines where $f \tau_{\text {hop }}<1$ faster relaxation thus means that the hopping is slower and the compound less conducting. Close to the magnetic transition temperature there is an additional increase in the relaxation rate and it diverges at $T_{c}$, while at low temperatures other processes like SN mechanism prevail.

\subsection{Line width}

The manganites $\mathrm{R}_{1-x} \mathrm{M}_{x} \mathrm{MnO}_{3}$ are very inhomogeneous systems, not only two different types of ions enter the same sublattice, but very often several distinct phases coexist in the same compound. As a result the NMR spectra of ${ }^{55} \mathrm{Mn}$ in these systems are inhomogeneously broadened. Octahedrally co-ordinated $\mathrm{Mn}^{3+}$ ion is known to be sensitive to its environment - this is a consequence of a strong vibronic interaction (Jahn-Teller effect) and not completely quenched orbital momentum. We expect therefore the $\mathrm{Mn}^{3+}$ line to be broader compared to the $\mathrm{Mn}^{4+}$ one. The line width of the motionally narrowed line is correlated with the disorder in the (R,M) sublattice which is documented in Table taken from Ref. [6].

TABLE

Width $\Delta_{1 / 2}$ at half the amplitude of ${ }^{55} \mathrm{Mn}$ NMR motionally narrowed line in several manganites containing $30 \%$ of $\mathrm{Mn}^{4+}$ ions. $\sigma^{2}$ characterizes the disorder caused by the difference of ionic radii of the lanthanoid and the substitution. $\sigma^{2}=\sum y_{i} r_{i}^{2}-$ $\left(\sum y_{i} r_{i}\right)^{2}$, where $r_{i}$ are the ionic radii and $y_{i}$ are corresponding fractional occupancies.

\begin{tabular}{l|c|c}
\hline \hline Composition & $\sigma^{2}\left[\AA^{2}\right]$ & $\Delta_{1 / 2}[\mathrm{MHz}]$ \\
\hline $\mathrm{La}_{0.7} \mathrm{Ca}_{0.3} \mathrm{MnO}_{3}$ & 0.0003 & 20 \\
$\mathrm{La}_{0.85} \mathrm{Na}_{0.15} \mathrm{MnO}_{3}$ & 0.0005 & 21 \\
$\mathrm{La}_{0.7} \mathrm{Sr}_{0.3} \mathrm{MnO}_{3}$ & 0.0019 & $20^{a}$ \\
$\mathrm{Pr}_{0.7} \mathrm{Ca}_{0.15} \mathrm{Sr}_{0.15} \mathrm{MnO}_{3}$ & 0.0022 & 26 \\
$\mathrm{La}_{0.7} \mathrm{~Pb}_{0.3} \mathrm{MnO}_{3}$ & 0.0038 & 34 \\
$\mathrm{Pr}_{0.7} \mathrm{Ba}_{0.3} \mathrm{MnO}_{3}$ & 0.0178 & 47 \\
\hline${ }_{\text {a }}$ average of $\Delta_{1 / 2}$ of compounds with Sr content 0.25 and 0.35
\end{tabular}

The line width of the ${ }^{55} \mathrm{Mn}$ NMR lines in manganites is of the order of several tens of $\mathrm{MHz}$. An eventual quadrupolar splitting, which should be present in the distorted Mn sites, is much smaller and can be thus neglected. If inequivalent Mn centers are present in the compound in question, the broadness of corresponding NMR lines often leads to their overlap which complicates the analysis of the spectra. 


\subsection{Enhancement factor and the amplitude of the spin-echo}

Similarly as the static field the rf field, besides direct coupling to the nuclear spins, couples also indirectly - it interacts with the electronic spin system and, through the hyperfine coupling, the time dependent part $b_{\mathrm{N}}(t)$ of the magnetic field on the nuclei is amplified. The amplification is very large in the ferromagnets and it is characterized by the "enhancement tensor" $\eta_{\alpha \beta}$. Enhancement tensor depends linearly on the local rf susceptibility $\chi_{\alpha \beta}$ of the electron spin system and on the hyperfine coupling tensor $A_{\alpha \beta}$ :

$$
\eta_{\alpha \beta}=\sum_{\gamma} A_{\alpha \gamma} \chi_{\gamma \beta}
$$

The local rf susceptibility is a complicated quantity. It is very large for the spins in the domain walls — rf field causes an oscillation of the domain wall and corresponding change of the perpendicular component of the electronic spins is large. For spins in the domains $\chi_{\alpha \beta}$ depends on the shape of the sample and on anisotropy field $B_{\mathrm{A}}$. Neglecting the anisotropy of hyperfine tensor, for a spherical sample and magnetization along the easy axis $z$ :

$$
\eta=\eta_{x x}=\eta_{y y}=B_{\mathrm{N}} /\left(B_{\text {ext }}+B_{\mathrm{A}}\right), \quad \eta_{z z}=0 .
$$

The exciting radiofrequency pulse should turn the nuclear magnetization by $\pi / 2$. With a fixed pulse duration $\delta_{t}$ this condition determines the optimal amplitude $b_{\mathrm{rf}}^{\text {opt }}$ of the radiofrequency field, which in turn depends on the enhancement factor $\eta[3]$ :

$$
b_{\mathrm{rf}}^{\mathrm{opt}}=\frac{h}{g_{\mathrm{N}} \mu_{\mathrm{N}}} \frac{1}{3 \eta \delta_{t}} .
$$

As the values of $\eta$ are different for nuclei in the domain walls and in the domains and, if there are several different phases present in the system, also for the nuclei in different phases, the $b_{\mathrm{rf}}^{\text {opt }}$ may be used to find out the region from which the spin-echo signal originates.

Providing that $b_{\mathrm{rf}}$ is fixed at its optimal value and that the correction for finite $\tau$ is made, the amplitude of the spin-echo signal is proportional to the number of resonating nuclei. This can be used to determine relative number of nuclei contributing to the spin-echo signal at different frequencies and therefore, after integration over corresponding NMR lines, also relative volume of different phases. Such information is qualitative only, however. Especially if comparing lines with very different resonance frequencies, other corrections of the amplitude may be important.

The amplitude of the spin-echo $\mathcal{A}$ decreases as the temperature is raised because the populations of the energy levels of the nuclei equalize. For thermal energy larger than the hyperfine splitting (typically for $T$ larger than $0.1 \mathrm{~K}$ ) $\mathcal{A} \sim 1 / T$ follows from the Boltzmann statistics. This statement is again qualitative, as the hyperfine splitting itself is temperature dependent and so is the enhancement factor $\eta$. 


\section{Magnetic phase transition}

NMR is very useful tool for the study of temperature dependence of the electronic magnetic moments as it probes their behavior locally through the hyperfine field (4). In ferromagnetic manganites the combination of NMR and DC magnetization studies are of particular importance when determining the nature of the magnetic transition. Due to the inhomogeneity of these systems, the ferromagnetic and paramagnetic phases may coexist below the temperature at which nonzero magnetization is observed. The comparison of NMR and magnetization data then allows to determine relative volumes of these phases, as well as to find out whether the transition is continuous or discontinuous.

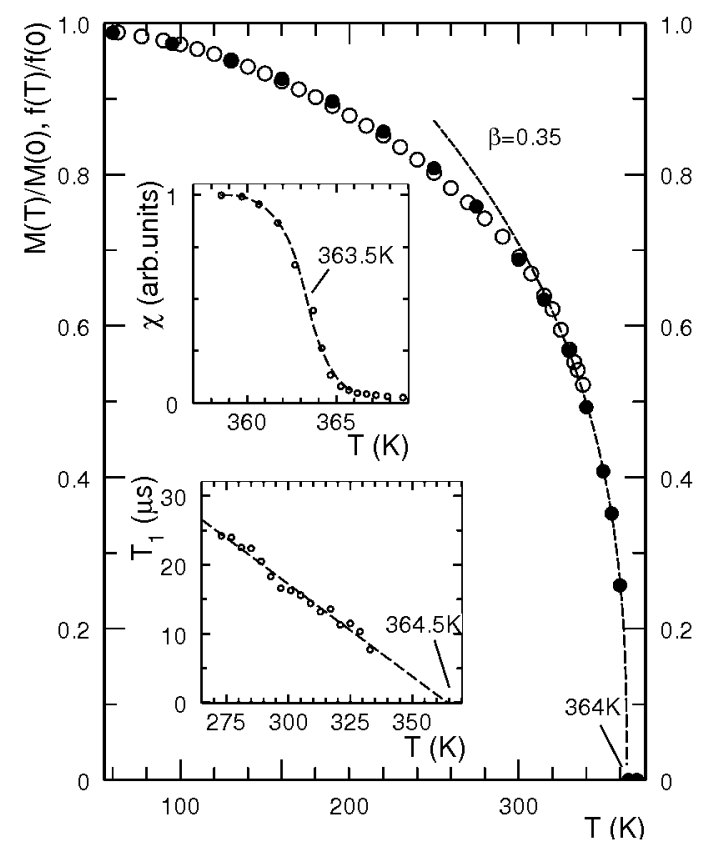

Fig. 2. The temperature dependence of reduced magnetization $(\bullet)$ and reduced NMR frequency (o) in $\mathrm{La}_{0.7} \mathrm{Sr}_{0.3} \mathrm{MnO}_{3}$. The dashed curve is the fit to the power law. In the lower inset the spin-lattice relaxation time vs. $T$ is displayed together with a linear fit (dashed line). In the upper inset the ac susceptibility vs. $T$ is shown.

Typical example of a continuous transition is found in $\mathrm{La}_{0.7} \mathrm{Sr}_{0.3} \mathrm{MnO}_{3}$ [4]. As seen from Fig. 2 temperature dependence of the reduced magnetization and reduced NMR frequency coincide in the whole temperature region where the spin-echo signal may be detected. Moreover, linear fit to $T_{1}$ in a critical region and the temperature dependence of the susceptibility give very similar values of $T_{\mathrm{c}}$.

More complex behavior is found in $\mathrm{Pr}_{0.7} \mathrm{Ca}_{0.15} \mathrm{Sr}_{0.15} \mathrm{MnO}_{3}$ and $\mathrm{Pr}_{0.7} \mathrm{Ba}_{0.3} \mathrm{MnO}_{3}$ compounds [6]. Here the temperature dependences of reduced 

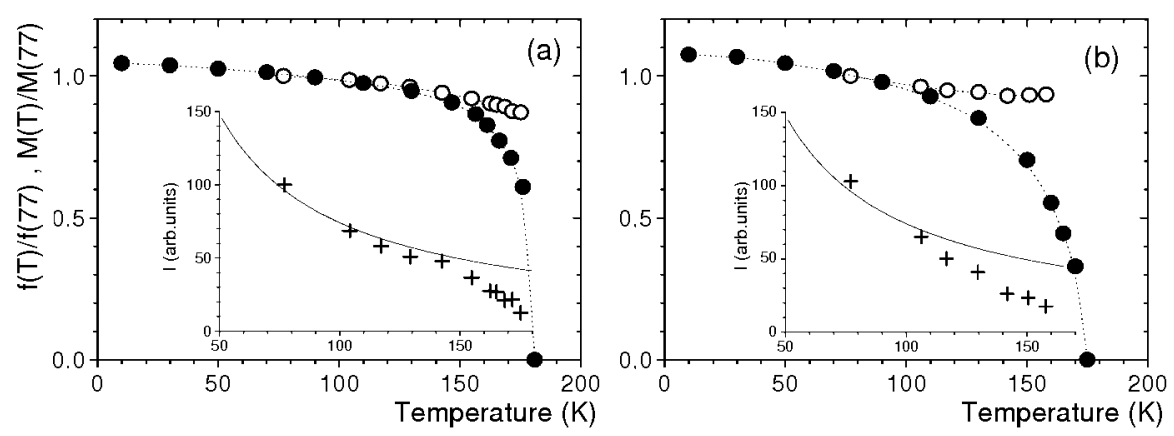

Fig. 3. $\mathrm{Pr}_{0.7} \mathrm{Ca}_{0.15} \mathrm{Sr}_{0.15} \mathrm{MnO}_{3}$ (a) and $\mathrm{Pr}_{0.7} \mathrm{Ba}_{0.3} \mathrm{MnO}_{3}$ (b). Temperature dependence of the NMR frequency $(0)$ and magnetization $(\bullet)$ relative to their values at $77 \mathrm{~K}$. The dotted curves serve as a guide for eyes only. In the insets the dependence of integrated intensity $I$ of the NMR line $(+)$ is displayed. The intensity was corrected for the spin-spin relaxation. The full curve is a hyperbolic dependence, expected in a single phase system.

magnetization and reduced NMR frequency differ - as $T_{\mathrm{c}}$ is approached $M(T) / M(77)$ decreases continuously to zero, while $f(T) / f(77)$ remains high (Fig. 3). This may be understood if the magnetic transition is of the first order and the volume of the ferromagnetic phase decreases for $T \rightarrow T_{\mathrm{c}}$. Such interpretation is further supported by the temperature dependence of the integrated intensity of the NMR signal (see insets in Fig. 3). As discussed above, in a single phase system this intensity is proportional to $1 / T$. In both systems studied a downward deviation from the $1 / T$ dependence is observed, reflecting the decreasing volume of the ferromagnetic phase.

\section{Co-existence of two ferromagnetic phases}

In Fig. 4a the NMR spectra of ${ }^{55} \mathrm{Mn}$ in $\mathrm{La}_{0.7} \mathrm{Ca}_{0.3} \mathrm{MnO}_{3}$ polycrystal at $T=200 \mathrm{~K}$, for several values of the pulse separation time $\tau$ are displayed. It is seen that with increasing $\tau$ the width of the spectra first decreases, but then remains constant after $\tau$ surpasses certain critical value. The simplest interpretation, that represents this dependence fairly well, is to assume that the NMR signal consists of two lines, which possess different $T_{2}$. For sufficiently large $\tau>14 \mu \mathrm{s}$ only the line possessing larger $T_{2}$ contributes and the spectra can be fitted by single Gaussian line $A_{1}$, centered at $322 \mathrm{MHz}$, with halfwidth of $22.4 \mathrm{MHz}$ and $T_{2}=6.86 \mu \mathrm{s}$. The contribution of faster relaxing nuclear spins can be then determined by subtracting $A_{1}(\tau)$ from the experimental spectrum (solid curves in Fig. 2b). This contribution can also be fitted by a Gaussian line $A_{2}$ centered at $307.3 \mathrm{MHz}$ with the same halfwidth as the line $A_{1}$, but shorter $T_{2}=4.1 \mu \mathrm{s}$. In Fig. 4c the mean relaxation time $T_{2}^{*}$ is displayed as function of the resonance frequency (the difference in $T_{2}\left(A_{1}\right)-T_{2}\left(A_{2}\right)$ is too small to resolve clearly the two 


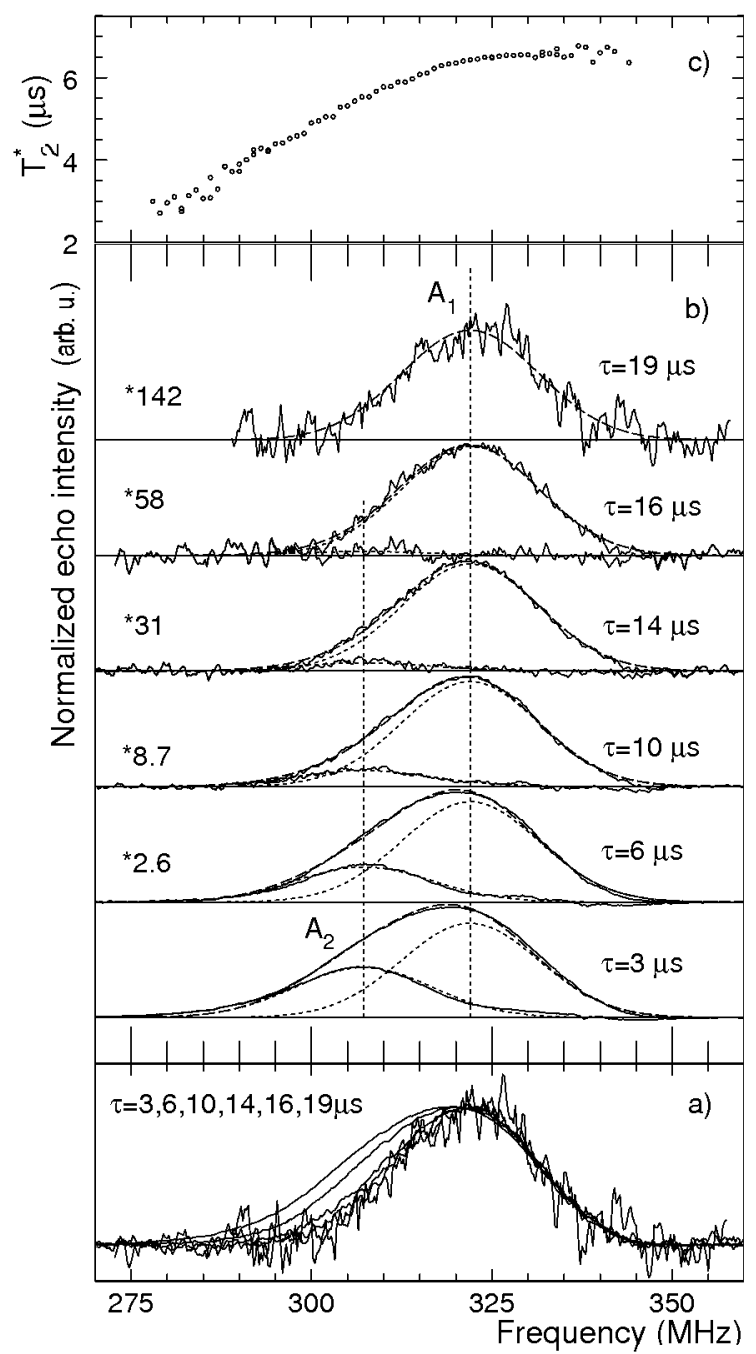

Fig. 4. $\mathrm{La}_{0.7} \mathrm{Ca}_{0.3} \mathrm{MnO}_{3}, T=200 \mathrm{~K}$. (a) NMR spectra for several values of $\tau$. (b) Decomposition of the spectra on two Gaussian lines $A_{1}, A_{2}$ (dotted curves). The full curve, corresponding to the $A_{2}$ line resulted from subtraction of $A_{1}$ line from the experimental spectrum. (c) Frequency dependence of the mean relaxation time $T_{2}^{*}$.

exponentials when measuring spin-echo decay at fixed frequency). The constant value of $T_{2}^{*}$ above $325 \mathrm{MHz}$ corresponds to the relaxation of the $A_{1}$ line, while the decrease in $T_{2}^{*}$ below $325 \mathrm{MHz}$ reflects the increase in the relative weight of nuclei possessing shorter $T_{2}$.

Two-line character of the NMR spectra suggests that the compound in question contains two different FM phases $A_{1}, A_{2}$, which give rise to the lines at higher and lower frequency, respectively. Phase $A_{2}$ is characterized by shorter $T_{2}$, 
i.e., faster dynamics of Mn nuclear spins that, according to (6) implies slower hopping of Mn electron holes. We have also found that the enhancement factor $\eta\left(A_{2}\right)$ is approximately twice smaller than $\eta\left(A_{1}\right)$, indicating larger magnetic anisotropy in the $A_{2}$ phase ( $c f$. Eq. (8)).

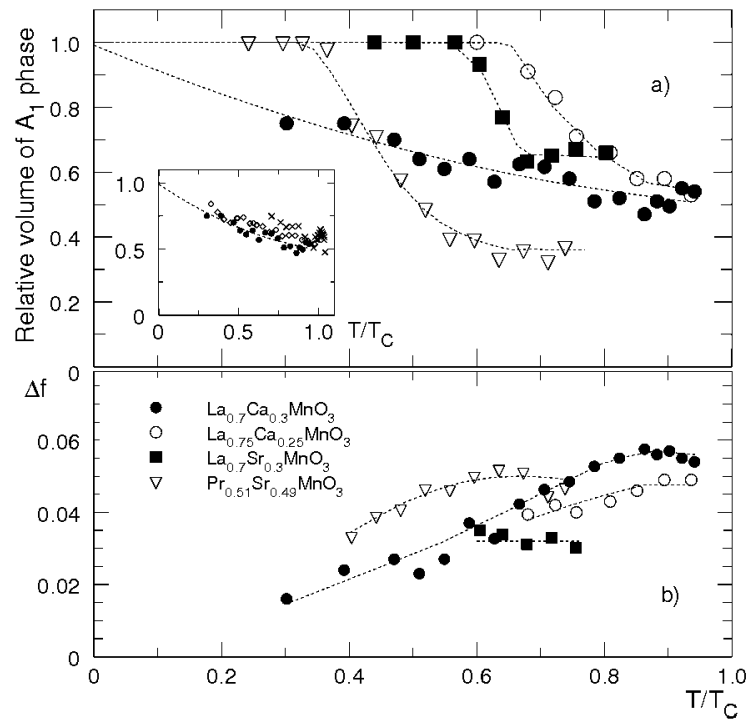

Fig. 5. Relative volume of the $A_{1}$ phase defined as $A_{1} /\left(A_{1}+A_{2}\right)$ (a) and relative difference of the resonance frequencies $\Delta f=2\left[f\left(A_{1}\right)-f\left(A_{2}\right)\right] /\left[f\left(A_{1}\right)+f\left(A_{2}\right)\right]$ (b) as functions of the reduced temperature for four different manganites. In the inset the NMR data for $\mathrm{La}_{0.7} \mathrm{Ca}_{0.3} \mathrm{MnO}_{3}(\bullet)$ are compared with the muon spin relaxation $(\times,[10])$ and the Mössbauer spectroscopy $(\diamond,[11])$. The data for (Pr,Sr) compound correspond to the FM phase only.

Using the approach described above the co-existence of the two different ferromagnetic phases $A_{1}, A_{2}$ was established in a number of other metallic-like manganites $[2,7]$, pointing to the fact that such phase separation is rather general phenomenon. In Fig. 5 the temperature dependence of the relative volume of the phase $A_{1}$ is displayed for four different manganites.

\section{Conclusions}

The nuclear magnetic relaxation of ${ }^{55} \mathrm{Mn}$ becomes fast for $T \rightarrow T_{\mathrm{c}}$ or when $f \tau_{\text {hop }} \rightarrow 1$ which makes detection of the spin-echo signal difficult and sometimes impossible. The NMR lines are inhomogeneously broadened and in many cases the spectra originating from inequivalent Mn ions overlap, making the analysis complicated and cumbersome. Despite these obstacles many valuable results were obtained. To the most recent results that, because of the space limitation, were 
not discussed above belong the study of the phase separation in the ferromagnetic, but insulating manganites [5,8] and the evidence for the charge modulated state in the $\mathrm{La}_{0.84} \mathrm{Sr}_{0.16} \mathrm{MnO}_{3}[9]$.

\section{Acknowledgments}

This paper is dedicated to the memory of Michail M. Savosta, who died tragically in February 2003, and without whom this paper would not be written. The work performed in the Institute of Physics, Prague was supported by the project AV0Z1-010-914 and by the grant 202/00/1601 of GA CR.

\section{References}

[1] T. Kubo, A. Hirai, H. Abe, J. Phys. Soc. Jpn. 26, 1094 (1969).

[2] M.M. Savosta, P. Novák, Phys. Rev. B 63, 226401 (2001).

[3] E.A. Turov, M.P. Petrov, Nuclear Magnetic Resonance in Ferro- and Antiferromagnets, Halsted, New York 1972.

[4] M.M. Savosta, V.A. Borodin, P. Novák, Phys. Rev. B 59, 8778 (1999).

[5] M.M. Savosta, V.I. Kamenev, V.A. Borodin, P. Novák, M. Maryško, J. Hejtmánek, K. Dörr, M. Sahana, Phys. Rev. B 67, 094403 (2003).

[6] M.M. Savosta, P. Novák, J. Hejtmánek, Z. Jirák, M. Maryško, Phys. Rev. Lett. 79, 4278 (1997).

[7] M.M. Savosta, V.A. Borodin, M. Maryško, Z. Jirák, J. Hejtmánek, P. Novák, Phys. Rev. B 65, 224418 (2002).

[8] P.A. Algarabel, J.M. De Teresa, J. Blasco, M.R. Ibarra, Cz. Kapusta, M. Sikora, D. Zajac, P.C. Riedi, C. Ritter, Phys. Rev. B 67, 134402 (2003).

[9] M.M. Savosta, P. Novák, Z. Jirák, Phys. Rev. B 67, 100411(R) (2003).

[10] R.H. Heffner, J.E. Sonier, D.E. MacLaughlin, G.J. Nieuwenhuys, G. Ehlers, F. Mezei, S.-W. Cheong, J.S. Gardner, H. Röder, Phys. Rev. Lett. 85, 3285 (2000).

[11] V. Chechersky, A. Nath, C. Michel, M. Hervieu, K. Ghosh, R.L. Greene, Phys. Rev. B 62, 5316 (2000). 